\title{
SELETIVIDADE E EFICÁCIA DO HERBICIDA CLORANSULAM-METIL NO CONTROLE DE PLANTAS DANINHAS NA CULTURA DA SOJA
}

\author{
Julio Pedro Laca-Buendia ${ }^{1}$ e Miguel Aparecido Nisrala ${ }^{2}$
}

'EPAMIG/CTTP. Rua Afonso Ratto, 1301 (Mercês). Uberaba, MG 38060-040 juliolaca@epamiguberaba.com.br

${ }^{2}$ DEFESA. Rua 94, n 720 (Sumiramis). Capinópolis, MG 38360-000

\section{RESUMO}

O objetivo do trabalho foi avaliar a seletividade e a eficácia do herbicida cloransulam-metil isolado e em mistura com lactofen no controle de plantas daninhas latifoliadas anuais em pós-emergência total na cultura da soja (cv. Doko), em área de Cerrado. Os tratamentos utilizados foram: cloransulam-metil (20, 30 e $40 \mathrm{~g} / \mathrm{ha})$, chlorimuron-ethyl+lactofen (12,5+96,0 g/ ha), cloransulam-metil+lactofen (30+96 g/ha), testemunha capinada e testemunha sem capina. Cloransulam-metil, independentemente das doses utilizadas, proporcionou eficiente controle de Acanthospermun hispidum (carrapicho-de-carneiro) e Blainvillea latifolia (erva-palha); em mistura com lactofen apresentou eficiente controle da Nicandra physaloides (joá-decapote). A mistura em tanque chlorimuron-ethyl+lactofen apresentou eficiente controle de Acanthospermun hispidum, Blainvillea latifolia e Nicandra physaloides. Não foram observadas diferenças significativas entre as produtividades nos diversos tratamentos químicos, mas o rendimento foi $48 \%$ menor na testemunha sem capina e 35\% menor no tratamento com cloransulam-metil na dose mais baixa, em relação a testemunha capinada. A aplicação dos tratamentos não afetou significativamente a população inicial e final, a altura da planta e a altura de inserção da primeira vagem. O índice de colheita mecânica foi excelente nos tratamentos químicos, exceto para cloransulam-metil a $20 \mathrm{~g} / \mathrm{ha}$, que foi médio. Todos os tratamentos testados foram seletivos para a soja cultivar Doko.

Palavras-chave: Acanthospermun hispidum, Blainvillea latifolia, controle químico, Glycine max, Nicandra physaloides.

\section{ABSTRACT \\ Efficacy and selectivity of cloransulam-methyl in postemergence control of weeds in soybean}

An experiment was carried out on a "Cerrado" oxisol with a loamy texture during 1996/97. The objective of present work was to evaluate the selectivity and efficacy of cloransulam-methyl, alone or tank mixed with lactofen, applied postemergence on soybean (cv. Doko) in controlling broadleaf weeds. The experimental design was a complete randomized block with seven treatments and four replications. The treatments were: cloransulam-methyl (20, 30 and $40 \mathrm{~g} / \mathrm{ha})$, chloransulammethyl+lactofen $(30+96 \mathrm{~g} / \mathrm{ha})$, chlorimuron-ethyl+lactofen $(12.5+96.0 \mathrm{~g} / \mathrm{ha})$ and control with and without weeds. Cloransulammethyl, independent of the used dose, provided efficient control of Acanthospermun hispidum and Blainvillea latifolia and in mixture with lactofen presented efficient control of Nicandra physaloides. The tank misture chlorimuron-ethyl+lactofen presented efficient control of Acanthospermun hispidum, Blainvillea latifolia and Nicandra physaloides. Significant differences were not observed among the productivity with the chemical treatments, but reduced productivity of the control without weed clearance and with lower dose of cloransulam-methyl in $48 \%$ and $35 \%$ in relation to the weed free control, respectively. The application of the treatments did not affect significantly the initial and final population, height of the plant and nor 
height of insert of the first bean. The index of mechanical harvesting with the chemical treatments was excellent, except for plots which received cloransulam-methyl at $20 \mathrm{~g} / \mathrm{ha}$. All the tested treatments were selective for soybean.

Key words: Acanthospermun hispidum, Blainvillea latifolia, chemical control, Glycine max, Nicandra physaloides.

\section{INTRODUÇÃO}

Em condições de Cerrado, no Triângulo Mineiro, o melhor rendimento tem sido obtido quando se mantem a cultura livre da competição das plantas daninhas durante os primeiros 40 dias após a emergência (Maia et al., 1992).

Herbicidas aplicados em pós-emergência na cultura da soja tem sido estudado por vários pesquisadores, conforme pode ser observado pelos trabalhos desenvolvidos por Pereira \& Bazoni (1995), Barros (1997a e 1997b), Constantin et al. (1997), Laca-Buendia \& Lara (1997), Laca-Buendia \& Nisrala (1997), Melhorança \& Souza (1997), Osipe \& Meyering (1997), Osipe et al. (1997), Rubin \& Kruse (1997) e Souza et al. (1997).

O objetivo do presente trabalho foi avaliar a seletividade e a eficácia do herbicida cloransulam-metil isolado e em mistura com lactofen no controle de plantas daninhas latifoliadas anuais, em pós-emergência total na cultura de soja, em área de Cerrado.

\section{MATERIAL E MÉTODOS}

O ensaio foi instalado no município de Capinópolis, MG, no ano agrícola 1996/97, em um Latossolo VermelhoEscuro, de textura argilosa, contendo $0,89 \%$ de matéria orgânica e pH(água)= 5,7.

A cultivar utilizada foi 'Doko', semeada em 10/12/ 96, utilizando-se o espaçamento de 0,50 m entre linhas, com semeadura manual, na densidade de 20 a 22 sementes por metro. A adubação de plantio foi feita usando-se $150 \mathrm{~kg} / \mathrm{ha}$ da fórmula 2:20:20 NPK.
O ensaio foi instalado com delineamento experimental de blocos casualizados, com quatro repetições, em parcelas de 2,0 $\mathrm{m} \times 7,0 \mathrm{~m}=14 \mathrm{~m}^{2}$, sendo colhidas as duas fileiras centrais, deixando-se $0,5 \mathrm{~m}$ em cada uma das extremidades, constituindo uma área útil de $1,0 \mathrm{~m} \times 5,0 \mathrm{~m}=5 \mathrm{~m}^{2}$. Os tratamentos utilizados estão descritos na Tabela 1.

Os herbicidas foram aplicados em 10/12/97, quando a soja apresentava o segundo trifólio e as plantas daninhas com 2 a 4 folhas. Foi utilizado um pulverizador costal pressurizado a $\mathrm{CO}$, dotado de barra com quatro pontas do tipo leque 110.04 , espaçados entre si de $0,5 \mathrm{~m}$ e a $0,5 \mathrm{~m}$ do solo, operando a uma pressão de $4,5 \mathrm{~kg} / \mathrm{cm}^{2}$, com consumo de calda de $256 \mathrm{l} /$ ha. As aplicações foram feitas entre $17 \mathrm{~h}$ e $17 \mathrm{~h} 20$, com temperatura do ar de $28^{\circ} \mathrm{C}$ e umidade relativa do ar de $70 \%$. O céu apresentava-se nublado, com ventos moderados e o solo úmido. As precipitações ocorridas durante a realização do experimento estão discriminadas na Tabela 2.

Para o controle das plantas daninhas na testemunha capinada foram necessárias três capinas manuais realizadas em 25/01/97, $10 / 02 / 97$ e 27/02/97. Foram realizadas duas aplicações com Thiodan CE, na dose de 0, 250 l/ha, em 12/ 02/97, para o controle de lagarta-das-folhas (Anticarsia gemmatalis) e em 20/03/97 para o controle de percevejos (Nezara virudula e Euschistus heros), na dose de 1,0 L/ha.

Foram realizadas avaliações visuais de toxicidade às plantas de soja, aos 7 e 15 dias após a aplicação, utilizandose a escala da EWRC, em que $1=$ sem injúria e 9= morte da planta.

Aos 15,21 e 30 dias após a aplicação, foram feitas avaliações visuais da eficácia do controle às plantas daninhas presentes, utilizando-se a escala percentual (0\% a 100\%), na área útil de cada parcela. Na testemunha sem capina foi realizada a contagem de todos os indivíduos por espécie das

Tabela 1. Tratamentos utilizados no experimento. Capinópolis, MG, 1996/1997.

\begin{tabular}{|c|c|c|c|c|}
\hline \multirow[b]{2}{*}{ Tratamento } & \multicolumn{2}{|c|}{ Doses do produto } & \multirow[b]{2}{*}{$\begin{array}{c}\text { Nome } \\
\text { comercial }\end{array}$} & \multirow[b]{2}{*}{$\begin{array}{c}\text { Concentração } \\
(\mathrm{g} / \mathrm{L} \text { ou } \mathrm{g} / \mathrm{kg})\end{array}$} \\
\hline & $\begin{array}{l}\text { Técnico } \\
\text { (g/ha) }\end{array}$ & $\begin{array}{l}\text { Comercial } \\
\text { (g ou L/ha) }\end{array}$ & & \\
\hline Cloransulam -metil ${ }^{(1)}$ & 20 & 23,8 & Pacto & 840 \\
\hline Cloransulam -metil ${ }^{(1)}$ & 30 & 35,7 & Pacto & 840 \\
\hline Cloransulam -metil ${ }^{(1)}$ & 40 & 47,6 & Pacto & 840 \\
\hline Chlorim uron - ethyl+lactofen & $12,5+96$ & $50,0+400$ & Classic+ Cobra & $250+240$ \\
\hline Cloransulam -metil+ lactofen & $30+96$ & $35,7+400$ & Pacto+Cobra & $840+240$ \\
\hline Testem unh a capinada & -- & -- & -- & --- \\
\hline Testemunha sem capina & --- & --- & -- & -- \\
\hline
\end{tabular}

(1) Foi adicionado o espalhante adesivo Agral a $0,2 \% \mathrm{v} / \mathrm{v}$. 
Seletividade e eficácia de cloransulam-metil em soja

Tabela 2. Precipitações, em mm, ocorridas durante a condução do ensaio. Capinópolis, MG, 1996/1997.

\begin{tabular}{|c|c|c|c|c|}
\hline Mês & $1^{0}$ Decêndio & $2^{0}$ Decêndio & $3^{0}$ Decêndio & Total \\
\hline Outubro & 12 & 0 & 75 & 87 \\
\hline Novem bro & 76 & 62 & 339 & 477 \\
\hline Dezem bro & 10 & 74 & 134 & 218 \\
\hline Janeiro & 345 & 104 & 234 & 683 \\
\hline Fevereiro & 30 & 36 & 40 & 106 \\
\hline Março & 140 & 40 & 40 & 220 \\
\hline Abril & 52 & 36 & 18 & 106 \\
\hline Total & & & & 1897 \\
\hline
\end{tabular}

plantas daninhas de maior abundância dentro de cada parcela, numa área de $1,0 \mathrm{~m}^{2}$.

Antes da colheita foi realizada uma avaliação visual do índice de facilidade de colheita mecânica, segundo a escala: 1- impossível (81 a 100\% de infestação), 2- alta infestação (61 a 80\% de infestação), 3- infestação razoável (41 a $60 \%$ de infestação), 4- boa colheita (21 a 40\% de infestação) e 5excelente (ótima) (0 a 20\% de infestação).

Quando as plantas da soja atingiram a fase de maturação dos grãos foi realizada a colheita (em 17 e 18/04/ 97), quando se realizou a pesagem dos grãos da área útil e contou-se o número de indivíduos $/ 5 \mathrm{~m}^{2}$ de cada parcela. A altura média das plantas e a altura de inserção da primeira vagem foram avaliadas em 10 plantas, ao acaso, dentro das duas fileiras centrais de cada parcela. Foram escolhidas, também ao acaso, 100 sementes em cada parcela, para determinação do peso dessas 100 sementes.

\section{RESULTADOS E DISCUSSÃO}

As espécies daninhas encontradas na área experimental foram: Acanthospermum hispidum (carrapicho-de-carneiro), com 16 indivíduos $/ \mathrm{m}^{2}$, Blainvillea latifolia (erva-palha), com 13 indivíduos $/ \mathrm{m}^{2}$ e Nicandra physaloides (mudar-decapote), com 70 indivíduos $/ \mathrm{m}^{2}$.

Para a densidade de plantas inicial e final, rendimento de grãos, altura da planta, altura de inserção da primeira vagem e do peso de 100 sementes, não foram detectadas diferenças entre os herbicidas e respectivas misturas estudadas com as testemunhas (Tabelas 3 e 4).

Nenhum dos herbicidas e misturas utilizadas apresentou efeitos de toxicidade nas plantas da soja, observando-se nota 1,0 (sem injúria) a 2,8 (traços), através da escala visual da EWRC (1 a 9) (Tabela 4).

Tabela 3. Resultados médios obtidos na avaliação da eficácia e seletividade do cloransulam-metil, em pós-emergência, para o controle de plantas daninhas latifoliadas anuais na cultura da soja, em áreas de Cerrado. Capinópolis, MG, $1996 / 1997$.

\begin{tabular}{|c|c|c|c|c|c|}
\hline \multirow[b]{2}{*}{ Tratamento } & \multirow{2}{*}{$\begin{array}{l}\text { Doses } \\
\text { (g/ha) }\end{array}$} & \multicolumn{2}{|c|}{ Numero de planta } & \multirow{2}{*}{$\begin{array}{l}\text { Produtividade } \\
\text { (kg/ha) }\end{array}$} & \multirow{2}{*}{$\begin{array}{l}\text { Altura da } \\
\text { planta } \\
(\mathbf{c m})\end{array}$} \\
\hline & & Inicial $^{(1)}$ & Final $^{(2)}$ & & \\
\hline Cloransulan-metil ${ }^{(3)}$ & 20 & 106,5 & 217,0 & 884 & 61,75 \\
\hline Cloransulan-metil ${ }^{(3)}$ & 30 & 115,5 & 227,8 & 2281 & 60,00 \\
\hline Cloransulan-metil ${ }^{(3)}$ & 40 & 114,5 & 218,5 & 2374 & 57,25 \\
\hline Chlorimuron-ethyl+lactofen & $12,5+96$ & 109,5 & 210,5 & 2099 & 54,75 \\
\hline Cloransulan-metil+lactofen & $30+96$ & 103,0 & 202,0 & 2024 & 60,00 \\
\hline Testemunha capinada & - & 117,5 & 237,0 & 2549 & 62,50 \\
\hline Testemunha sem capina & - & 111,5 & 210,2 & 1720 & 61,75 \\
\hline Média Geral & - & 111,1 & 217,6 & 2133 & 59,71 \\
\hline Valor de F & & $0,75 \mathrm{NS}$ & $0,73 \mathrm{NS}$ & $2,22 \mathrm{NS}$ & $0,74 \mathrm{NS}$ \\
\hline C.V.(\%) & & 10,8 & 12,6 & 18,2 & 10,9 \\
\hline
\end{tabular}

\footnotetext{
(1) Contagem realizada aos 15 dias após a aplicação, numa área de 3,0 $\mathrm{m}^{2}$.

(2) Contagem numa área de $7,0 \mathrm{~m}^{2}$.

${ }^{(3)}$ Foi adicionado o espalhante adesivo Agral a 0,2\%v/v.
} 
Tabela 4. Resultados médios obtidos na avaliação da eficácia e seletividade do cloransulam-metil, em pós-emergência para o controle de plantas daninhas latifoliadas anuais na cultura da soja, em área de Cerrado. Capinópolis, MG, 1996/ 1997.

\begin{tabular}{|c|c|c|c|c|c|c|}
\hline \multirow{2}{*}{ Tratam en to } & \multirow{2}{*}{$\begin{array}{l}\text { Doses } \\
(\mathrm{g} / \mathrm{ha})\end{array}$} & \multirow{2}{*}{$\begin{array}{c}\text { Inserção } \\
\text { da } \\
1^{2} \text { vagem } \\
\text { (cm) }\end{array}$} & \multirow{2}{*}{$\begin{array}{c}\text { Pesode } \\
100 \\
\text { sem entes } \\
\text { (g) }\end{array}$} & \multirow{2}{*}{$\begin{array}{c}\text { Facilid ade } \\
\text { de } \\
\text { Colh eita } \\
(1 \text { a } 5)\end{array}$} & \multicolumn{2}{|c|}{$\begin{array}{l}\text { Fitotoxicidade } \\
\text { EWRC (1 a 9) }\end{array}$} \\
\hline & & & & & $7 \mathbf{D A A}$ & 15D A A \\
\hline Cloransulam -m etil(2) & 20 & 19,5 & 13,1 & $3,5 b^{(1)}$ & $1,0 \mathrm{~b}$ & $1,0 \mathrm{~b}$ \\
\hline Cloran sulam -m etil ${ }^{(2)}$ & 30 & 19,0 & 14,0 & $4,0 \mathrm{ab}$ & $1,0 \mathrm{~b}$ & $1,0 \mathrm{~b}$ \\
\hline Cloransulam -m etil(2) & 40 & 19,2 & 14,4 & $4,8 \mathrm{a}$ & $1,2 \mathrm{~b}$ & $1,0 \mathrm{~b}$ \\
\hline Chlorimuron-ethyl+ lactofen & $12,5+96$ & 17,2 & 13,8 & $4,5 \mathrm{ab}$ & $2,8 \mathrm{a}$ & $1,8 \mathrm{a}$ \\
\hline Cloransulam -m etil+ lactofen & $30+96$ & 19,8 & 14,2 & $4,8 \mathrm{a}$ & $2,8 \mathrm{a}$ & $1,5 \mathrm{a}$ \\
\hline Testem unh a capin ada & - & 18,8 & 13,8 & $5,0 \mathrm{a}$ & $1,0 \mathrm{~b}$ & $1,0 \mathrm{~b}$ \\
\hline Testem unh a sem capin a & - & 17,2 & 13,8 & $1,2 \mathrm{c}$ & $1,0 \mathrm{~b}$ & $1,0 \mathrm{~b}$ \\
\hline Média Geral & - & 18,7 & 13,9 & 3,9 & 1,5 & 1,2 \\
\hline Valor de F & & $0,8 \mathrm{NS}$ & $1,3 \mathrm{NS}$ & $29,5 * *$ & $13,0 * *$ & $4,5 * *$ \\
\hline DMS & & - & - & 1,1 & 1,0 & 0,7 \\
\hline C.V. $(\%)$ & & 12,3 & 5,0 & 12,1 & 30,1 & 25,6 \\
\hline
\end{tabular}

(1) DAA=Dias após a aplicação.

(2) Foi adicionado o espalhante adesivo Agral a $0,2 \% \mathrm{v} / \mathrm{v}$.

(3) As médias, na mesma coluna, seguidas pela mesma letra, não diferem significativamente entre si pelo teste de Tukey ao nível de $5 \%$ de probabilidade.

Tabela 5. Controle percentual de plantas daninhas na cultura da soja. Capinópolis, MG, 1996/1997.

\begin{tabular}{|c|c|c|c|c|c|c|c|}
\hline \multirow[t]{2}{*}{ Tratamento } & \multirow{2}{*}{$\begin{array}{l}\text { Dose } \\
\text { (g/ha) }\end{array}$} & \multicolumn{2}{|c|}{$\begin{array}{c}\text { Acanthospermum } \\
\text { hispianm }^{(1)}\end{array}$} & \multicolumn{2}{|c|}{$\begin{array}{l}\text { Blainvillea } \\
\text { latifolia }{ }^{(1)}\end{array}$} & \multicolumn{2}{|c|}{$\begin{array}{c}\text { Nicandra } \\
\text { physaloides }\end{array}$} \\
\hline & & $15 \mathrm{DAA}$ & $30 \mathrm{DAA}^{(3)}$ & $15 \mathrm{DAA}$ & $30 \mathrm{DAA}$ & $15 \mathrm{DAA}$ & $30 \mathrm{DAA}$ \\
\hline Cloransulan-metil ${ }^{*}$ & 20 & $98 \mathrm{~b}^{(2)}$ & 99 & $99 b^{(2)}$ & $98 \mathrm{ab}$ & $52,0 c^{(2)}$ & $47,8 \mathrm{e}$ \\
\hline Cloransulan-metil(*) & 30 & $99 \mathrm{~b}$ & 99 & $99 \mathrm{~b}$ & $99 \mathrm{ab}$ & $73,0 \mathrm{~b}$ & $69,0 \mathrm{~d}$ \\
\hline Cloransulan-metil $\left.{ }^{*}\right)$ & 40 & $99 \mathrm{~b}$ & 99 & $99 \mathrm{~b}$ & $98 \mathrm{~b}$ & $76,5 b$ & $77,5 \mathrm{c}$ \\
\hline $\begin{array}{l}\text { Chlorimuron -ethyl+ } \\
\text { lactofen }\end{array}$ & $12,5+96$ & $99 \mathrm{~b}$ & 99 & $98 \mathrm{~b}$ & $96 \mathrm{~b}$ & $98,8 \mathrm{a}$ & $96,5 b$ \\
\hline $\begin{array}{l}\text { Cloransulan-metil+ } \\
\text { lactofen }\end{array}$ & $30+96$ & $99 \mathrm{~b}$ & 99 & $98 \mathrm{~b}$ & $96 b$ & $99,0 \mathrm{a}$ & $97,0 \mathrm{~b}$ \\
\hline Testemunha capinada & - & $100 \mathrm{a}$ & 100 & $100 \mathrm{a}$ & $100 \mathrm{a}$ & $100,0 \mathrm{a}$ & $100,0 \mathrm{a}$ \\
\hline Testemunha sem capina & - & $0 c$ & 0 & $0 c$ & $0 \mathrm{c}$ & $0,0 \mathrm{~d}$ & $0,0 \mathrm{f}$ \\
\hline $\mathrm{N}^{0}$ de individuos $/ \mathrm{m}^{2}$ & - & 16 & 16 & 13 & 17 & 70,0 & 120,0 \\
\hline Valor de F & - & $9999 * *$ & - & $1778,1^{* *} *$ & $314,1^{* *}$ & $397,9 * *$ & $807,2 * *$ \\
\hline C. V. $(\%)$ & & 0,6 & & 2,1 & 5,0 & 5,2 & 3,6 \\
\hline \multicolumn{8}{|c|}{$\begin{array}{l}\text { Dados transformados em Arc.seno } \sqrt{\mathrm{x} / 100} \text { para realização da análise da variância. } \\
\text { DAA= dias após a aplicação. } \\
\text { Foi adicionado o espalhante adesivo Agral a } 0,2 \% \mathrm{v} / \mathrm{v} \text {. } \\
\text { As médias na mesma coluna seguidas pela mesma letra, não diferem estatisticamente entre si, pelo teste de Tukey ao nível de } 5 \% \text { de } \\
\text { probabilidade. }\end{array}$} \\
\hline
\end{tabular}

Na avaliação visual para facilidade de colheita mecânica, através do uso de escala de 1 a 5 , verificou-se que o único herbicida que não apresentou diferença com releção à testemunha capinada, foi cloransulam-metil a $30 \mathrm{~g} / \mathrm{ha}$, sendo que nas outras doses testadas e com as misturas de lactofen não houve diferença em relação à testemunha capinada (Tabela 4).

Para o controle do carrapicho-de-cameiro e erva-palha, verificou-se que cloransulam-metil e as misturas cloransulam-metil+lactofen e chlorimuron-ethyl+lactofen 
apresentaram excelente controle para estas espécies daninhas, com índices de controle de 96 a 99\%, até 30 dias após a aplicação (Tabela 5). Para joá-de-capote, o cloransulam-metil, nas doses testadas, apresentou um controle não satisfatório (52 a $77 \%$ aos 15 DAT), sendo que em mistura com lactofen, assim como a mistura de chlorimuron-ethyl+lactofen, apresentaram índices de controle acima de $98 \%$, até 30 dias após a aplicação (Tabela 5).

\section{CONCLUSÕES}

Cloransulam-metil, a 20, 30 e $40 \mathrm{~g} / \mathrm{ha}$, apresentou eficiente controle de Acanthospermun hispidum (carrapicho-decarneiro) e Blainvillea latifolia (erva-palha). Clorasulammetil+lactofen, a 30+96 g/ha, demonstrou excelente controle de Nicandra physaloides (joá-de-capote). Chlorimuronethyl+lactofen, a 12,5+96,0 g/ha, apresentou eficiente controle de Acanthospermun hispidum (carrapicho-de-cameiro), Blainvillea latifolia (erva-palha) e Nicandra physaloides (joáde-capote).

Todos os herbicidas apresentaram seletividade para a cultura da soja, quando utilizados em área total, em pós-emergência.

\section{LITERATURA CITADA}

BARROS, A. C. de. Avaliação da eficiência e da seletividade dos herbicidas diclosulam, cloransulam-metil em comparação com flumetsulatn e chlorimuron-etil+lactofen para o controle de plantas invasoras de soja. ln: CONGRESSO BRASILEIRO DA CIÊNCIA DAS PLANTAS DANINHAS, 21. Caxambu, MG, 1997. Resumos... Viçosa, MG: SBCPD, 1997a. p. 52.

BARROS, A. C. de. Cloransulam-metil aplicado em pósemergência para o controle de plantas daninhas infestantes na cultura da soja. ln: CONGRESSO BRASILEIRO DA CIÊNCIA DAS PLANTAS DANINHAS, 21. Caxambu, MG, 1997. Resumos... Viçosa, MG: SBCPD, 1997b. p. 53.

CONSTANTIN, J.; ZAGATTO, A.; CONTIERO, R..; ITA, A. 0. Eficiência e seletividade do herbicida cloransulammetil no controle de plantas daninhas latifoliadas na cultura da soja (Glycine max (L.) Merril). In. CONGRESSO BRASILEIRO DA CIÊNCIA DAS PLANTAS DANINHAS, 21. Caxambu, MG, 1997. Resumos... Viçosa, MG: SBCPD, 1997. p. 71.

LACA-BUENDIA, J. P.; LARA, J. F. R. Eficácia e seletividade do oxasulfuron, aplicado na pós-emergência, para o controle de plantas daninhas latifoliadas anuais na cultura da soja (Glycine $\max$ (L.) Merril). In: CONGRESSO
BRASILEIRO DA CIÊNCIA DAS PLANTAS DANINHAS, 21. Caxambu, MG, 1997. Resumos... Viçosa, MG: SBCPD, 1997. p. 101.

LACA-BUENDIA, J. P.; NISLARA, M. A. Atividade biológica e seletividade do oxasulfuron, aplicado na pósemergência, para o controle de plantas daninhas latifoliadas anuais na cultura da soja (Glycine $\max$ (L.) Merril). In: CONGRESSO BRASILEIRO DA CIÊNCIA DAS PLANTAS DANINHAS, 21. Caxambu, MG, 1997. Resumos... Viçosa, MG: SBCPD, 1997. p. 103.

MAIA, A. C.; REZENDE, A. M. de; LACA-BUENDIA, J. P. del C. Período crítico de competição de uma comunidade natural de plantas daninhas com a cultura da soja (Glycine max (L.) Merrill), no Triângulo Mineiro. In: SEMINÁRIO NACIONAL DE PESQUISA DE SOJA, 2. Brasília, 1991. Anais... Londrina: EMBRAPACNPSo, 1992, p. 370-380. (EMBRAPA-CNPSo. Documentos, 1 )

MELHORANÇA, A. L.; SOUZA, J. A. Efeito do herbicida flumioxazin aplicado em pós-emergência no controle das plantas daninhas na cultura da soja. ln: CONGRESSO BRASILEIRO DA CIÊNCIA DAS PLANTAS DANINHAS, 21. Caxambu, MG, 1997. Resumos... Viçosa, MG: SBCPD, 1997. p. 111.

NETO, F. S.; ALMEIDA, F. L. S.; PASSIM, T. Eficácia de herbicidas no controle de plantas latifoliadas na cultura da soja. In: CONGRESSO BRASILEIRO DE HERBICIDAS E PLANTAS DANINHAS, 18. Londrina, 1991. Resumos... Londrina: SBHED, 1991, p. 47.

OSIPE, R.; MEYERING, R. Seletividade e controle de plantas daninhas através da aplicação do herbicida cloransulam-metil, em pós-emergência da cultura da soja (Glycine $\max (\mathrm{L}$.$) Merril). In: CONGRESSO BRASI-$ LEIRO DA CIÊNCIA DAS PLANTAS DANINHAS, 21. Caxambu, MG, 1997. Resumos... Viçosa, MG: SBCPD, 1997. p.114.

OSIPE, R.; NISI-UMURA, M; LOPES, D. Avaliação da eficiência e seletividade de herbicidas aplicados em misturas de tanque, em pós-emergência na cultura da soja. In: CONGRESSO BRASILEIRO DA CIÊNCIA DAS PLANTAS DANINHAS, 21. Caxambu, MG, 1997. Resumos... Viçosa, MG:SBCPD, 1997. p. 115.

PEREIRA, F. de A. R.; BAZONI, R. Avaliação de herbicidas na cultura da soja em áreas de Cerrado de Mato Grosso do Sul. Campo Grande, MS. EMPAER, 1995. 38p. (EMPAER-MS. Documentos, 45). 
RUBIN, R.; KRUSE, N. D. Avaliação de eficiência agronômica e seletividade do herbicida cloransulam-metil, aplicado em pós-emergência, no controle de Xanthium strumarium, na cultura da soja. In: CONGRESSO BRASILEIRO DA CIÊNCIA DAS PLANTAS DANINHAS, 21. Caxambu, MG, 1997. Resumos... Viçosa, MG: SBCPD, 1997. p. 130.
SOUZA, I. F.; ARGENTA, J. A. ; BARROSO, A. L. L. Misturas de herbicidas de pós-emergência para o controle de espécies de folhas largas em soja. In: CONGRESSO BRASILEIRO DA CIÊNCIA DAS PLANTAS DANINHAS, 21. Caxambu, MG, 1997. Resumos... Viçosa, MG: SBCPD, 1997. p. 136. 